\section{Oscillator Strengths of the Ultraviolet $\gamma$ Bands of NO*}

\author{
S. S. PENNER \\ Daniel and Florence Guggenheim Jet Propulsion Center, California \\ Institute of Technology, Pasadena, California
}

(Received November 25, 1959)
$I^{N}$ $\mathrm{N}$ a recent paper, Erkovich, ${ }^{1}$ after examining experimental data by Marmo, ${ }^{2}$ Mayence, ${ }^{3}$ and Weber and Penner, ${ }^{4}$ comes to the conclusion that the effective electronic $f$ number for the $\gamma$ bands of $\mathrm{NO}$ is 0.043 . This value is roughly 17 times larger than the $f$ number estimates obtained by Weber and Penner ${ }^{4}$ and by Bethke. $^{5}$

The analysis of Erkovich is based on the assumption that quantitative intensity data can be obtained from a study of low. pressure experimental results obtained under conditions in which the experimental slitwidth is very large compared with the linewidth, without direct quantitative measurement of the experimental slit function. The dangers involved in the use of this pro. cedure are too well known to require elaboration. ${ }^{6-8}$ For this reason, we content ourselves with brief summary statements of those parts of the analysis performed by Erkovich that require serious reexamination.

(1) The experimental slit function is generally not related in a simple manner to the estimated instrumental bandwidth. It should be measured directly even if only semiquantitative data are desired.

(2) The rotational lines of the NO bands are indeed well separated at atmospheric pressure and at lower pressures. For this reason, significant pressure broadening must be achieved in order to remove the rotational fine structure. The value of the optical collision diameter assumed by Erkovich is based on the data of Thorson and Badger ${ }^{9}$ and depends directly on the $f$ number estimates of Weber and Penner since these were used for the computation of the line widths. Thus it is inconsistent to use the collision diameter of Thorson and Badger in conjunction with an $f$ number of 0.040 .

(3) Weber and Penner determined the $f$ number by performing two independent series of measurements, namely, studies using self-broadening at pressures between 60 and 400 psia and studies using inert-gas broadening at a pressure of 500 psia. ${ }^{4}$ Bethke's measurements involve inert-gas broadening and extend to pressures of 1000 psia. ${ }^{j}$ The collision diameters for self-broadening are expected to be larger than for inert-gas broadening in accord with the experimental results obtained for infrared vibration-rotation bands. ${ }^{10}$ The calculated absorption coefficient curve at $10 \mathrm{~atm}$ shown in Fig. 2 of Erkovich's paper exhibits surprisingly narrow lines; in any event, it is inapplicable to our high-pressure data at 400 and 500 psia and to Bethke's data which were obtained at pressures up to 1000 psia. Bethke's data on the pressure dependence of integrated absorption show very clearly that pressures in excess of $10 \mathrm{~atm}$ of argon are required for some of the bands before the integrated absorption ceases to increase rapidly with pressure.

(4) The remarkable fit to our experimental data obtained by Erkovich appears to involve the fortuitous cancellation of errors between an erroneous estimate for the $f$ number, an inconsistent value for the linewidth, and the invalid assumption that our data can be accounted for by using a formula that is applicable only if essentially no overlapping occurs between spectral lines. Furthermore, the calculations made by Erkovich required the use of a slit function for our experiments, the shape and width of which was certainly unknown to us.

Erkovich's conjecture concerning the equivalence of the $f$ numbers for the $\beta$ and $\gamma$ bands is not in accord with measured results. ${ }^{5,11}$ That the $\gamma$ bands near the bandhead are, in fact, well overlapped
TABLE I. $f$ Number estimates obtained for the $\mathrm{NO}_{i}$ bands by several investigators.

\begin{tabular}{lll}
\hline \multicolumn{1}{c}{ Author(s) } & Method & \multicolumn{1}{c}{$f_{\gamma}$} \\
\hline Weber and Penner (1957) & $\begin{array}{c}\text { Self-broadening and } \\
\text { inert-gas broadening } \\
\text { Inert-gas broadening } \\
\text { Absolute intensity } \\
\text { meastrements behind } \\
\text { khocks in air } \\
\text { Keck } \text { high-resolution } \\
\text { spectroscopy }\end{array}$ & $0.0024 \pm 0.0005$ \\
$\begin{array}{c}\text { Dieke } t \text { al. preliminary } \\
\text { 1959) }\end{array}$ & $\begin{array}{c}0.00240 \pm 5 \% \\
\text { precision of the data } \\
\text { was not specified in } \\
\text { reference 11) }\end{array}$ \\
\hline
\end{tabular}

at $300^{\circ} \mathrm{K}$ and 500 psia of He may be verified by elementary considerations. The linewidth estimates of Thorson and Badger ${ }^{9}$ yielded an optical collision diameter of $3.8 \mathrm{~A}$ for the $\gamma$ bands of $\mathrm{NO}$ in mixtures of $\mathrm{N}_{2}$ and NO. ${ }^{9}$ Earlier linewidth measurements on the infrared vibration-rotation bands of $\mathrm{NO}$ correspond to optical collision diameters of $2.5 \mathrm{~A}$ for $\mathrm{NO}$-He mixtures and to 4.2 A for NO-NO mixtures. "Even assuming an optical collision diameter as small as $2.5 \mathrm{~A}$ for the $\gamma$ bands of NO in NO-He mixtures, we find that the collision semihalf-width at $300^{\circ} \mathrm{K}$ and 500 psia is about $2.5 \mathrm{~cm}^{-1}$. Near the bandhead the rotational spacing is about $3 \mathrm{~cm}^{-1}$. Cnder these conditions, it is easily shown (see, for example, Fig. 11-6 of reference 8), that the absorption coeffcient is a nicely continuous function of wave number, a result that is in good accord with the directly measured ${ }^{5}$ dependence of integrated absorption on pressure.

Estimates of / numbers for the electronic bands of NO have been made also on the basis of absolute intensity measurements behind shock fronts by Keck et al. ${ }^{2}$ For the wavelength range extending from 2500 to $2700 \mathrm{~A}$, Keck $e t a l$. found an effective $f$ number for the NO $\gamma$ bands of $0.001 \pm 0.0005$, which is roughly half as large as the room-temperature results published in the earlier papers. ${ }^{4,5}$ For the $\beta$ bands of NO, Keck et al. found $f=0.006 \pm 0.0002$ for the wavelength interval 3500 to $5000 \mathrm{~A}$, which is appreciably larger than Bethke's estimate. Keck et al. also quote preliminary results by Dieke and co-workers who found $f_{\gamma} \cong 0.0013$ using highresolution spectroscopy. A tabular summary of available $f$ number estimates for the NO $\gamma$ bands is given in Table I.

In conclusion, we wish to point out the important fact that a significant increase in the $f$ numbers of the No bands would imply a significant increase in radiant heat transfer to missile nose cones during reentry into the earth's atmosphere (see, for example, Chap. 14, reference 8).

\footnotetext{
* Supported by the Office of Naval Research, U. S. Navy.

S. P. Erkovich, Optika i Spektroskopia 6, 193 (1959).

F. F. Marmo, J. Opt. Soc, Am. 43, 1186 (1953)

s. Mayence, Ann. Physik 7, 453 (1952)

4 D. Weber and S. S. Penner, J. Chem. Phys. 26, 860 (1957).

G. W. Bethke, J. Chem. Phys. 31, 662 (1959).

E. C. Kemble, J. Chem. Phys. 3 , 316 (1935).

E. B. Wilson, Jr., and A. J. Wells. J. Chem. Phys. 14, 578 (1946).

\& S. S. Penner, Quantitatwe Molecular Spectroscopy and Gas Emissivilies Addison-Wesley Publishing Company, Reading, Massachusetts, 1959),

Chap. 6

W. R. Thorson and R. M. Badger, J. Chem, Phys. 27, 609 (1957)

${ }^{10}$ Reference 8, p. 211.

"1 This linewidth estimate should be regarded as a reasonably conservative lower limit. Kivel, Mayer, and Bethe [Ann. Phys. 2, 57 (1957)] have calculated a linewidth of about $1 \mathrm{~cm}^{-1}$ at normal density. 7, 1 J. C. Keck, J. C. Camm, B. Kivel, and T. Wentink, Jr., Ann. Phys.
} 